\title{
MALAT1 functions as a competing endogenous RNA to regulate SMAD5 expression by acting as a sponge for miR-142-3p in hepatocellular carcinoma
}

Qiangfeng $\mathrm{Yu}^{1,2}$, Leyang Xiang ${ }^{2}$, Zhanjun Chen ${ }^{2}$, Xincheng Liư ${ }^{3}$, Huohui Ou ${ }^{2}$, Jianyin Zhou ${ }^{*}$ and Dinghua Yang ${ }^{2^{*}}$

\begin{abstract}
Background: Long non-coding RNAs are involved in the pathology of various tumors, including hepatocellular carcinoma. The expression of metastasis-associated lung adenocarcinoma transcript 1 (MALAT1) is increased in numerous types of tumors and is involved in tumor cell proliferation, migration, invasion and apoptosis. MALAT1 level was reported to be upregulated in hepatocellular carcinoma tissues, but its roles and the specific molecular mechanisms are still unclear.

Methods: The expression of MALAT1 and miR-142-3p in hepatocellular carcinoma tissues, cell lines and adjacent non-tumor tissues was assessed by Q-PCR. The putative-binding sites between MALAT1 and miR-142-3p were predicted by bioinformatics analysis. The expression of MALAT1 in HepG2 and SMMC-7721 cells was knocked down by transfection with MALAT1 siRNAs. Cell viability was assessed by the Cell Counting Kit-8 (CCK-8) assay after the indicated transfection in HepG2 and SMMC-7721 cells. Cell proliferation was assessed by EdU assay, and cell apoptosis was explored by flow cytometry. The migration and invasion potency of HepG2 and SMMC-7721 cells was assessed by the cell migration assay and matrigel invasion assay. Protein level of vimentin, E-cadherin and SMAD5 were assessed by Western blot.

Results: Overexpressed MALAT1 acts as a competing endogenous RNA sponge for miR-142-3p in hepatocellular carcinoma. The knockdown of MALAT1 inhibited the proliferation, migration, invasion, and epithelial cell-to-mesenchymal transition (EMT), and promoted apoptosis of hepatocellular carcinoma cells via miR-142-3p. MiR-142-3p inhibited cell proliferation, migration, invasion and EMT, and promoted the cell apoptosis by targeting SMAD5 in hepatocellular carcinoma. MALAT1 promoted tumor growth by regulating the expression of miR-142-3p in vivo.
\end{abstract}

Conclusion: MALAT1 promoted cell proliferation, migration, and invasion of hepatocellular carcinoma cells by antagonizing miR-142-3p.

Keywords: MALAT1, miR-142-3p, Hepatocellular carcinoma, EMT, SMAD

\footnotetext{
*Correspondence: zhoujianyin2000@sina.com; dhyangyd@yahoo.com; yangdinghuadoctor@163.com

2 Department of Hepatobiliary Surgery, Nanfang Hospital Affiliated to Southern Medical University, Guangzhou, China

${ }^{4}$ Department of Hepatobiliary and Pancreatic Surgery, Zhongshan Hospital, Xiamen University, Xiamen, China

Full list of author information is available at the end of the article
} (http://creativecommons.org/licenses/by/4.0/), which permits unrestricted use, distribution, and reproduction in any medium, provided you give appropriate credit to the original author(s) and the source, provide a link to the Creative Commons license, and indicate if changes were made. The Creative Commons Public Domain Dedication waiver (http://creativecommons.org/ publicdomain/zero/1.0/) applies to the data made available in this article, unless otherwise stated. 


\section{Introduction}

As the second leading cause of cancer-related deaths worldwide, hepatocellular carcinoma has led to approximately 800,000 deaths and is associated with 850,000 new cases each year [1]. Hepatitis B and $C$ viral infection, intake of alcohol, and exposure to the fungal metabolite aflatoxin B1 are the main risk factors for hepatocellular carcinoma [2, 3]. Primary hepatocellular cancer is a type of heterogeneous tumor in which the tumors metastasizes to hepatocellular tissue from other sites. As the main primary hepatocellular cancer, hepatocellular carcinogenesis is multifactorial process with different susceptibility factors. Large regional variations in the prevalence of hepatocellular carcinogenesis have been reported $[4,5]$. As the most common primary liver cancer, hepatocellular carcinoma is heterogeneous in nature [6]. Many molecular and cell signaling pathways have been implicated in the development of hepatocellular carcinoma, and the progress of tumorigenesis in hepatocellular carcinoma remains unclear. Most important in the effort to improve survival in patients with hepatocellular carcinoma patients is the identification of therapeutic targets for the disease.

Long noncoding RNAs (lncRNAs) are a heterogeneous class of transcripts longer than 200 nucleotides with limited protein-coding ability but play an important role in the pathogenesis of hepatocellular carcinoma [7]. Some lncRNAs are aberrantly expressed in hepatocellular carcinoma or other human cancers where their interaction with various macromolecules such as DNA, chromatin, proteins, and RNAs affects cell proliferation, apoptosis, angiogenesis, invasion, and metastasis of hepatocellular carcinoma cells [8-10]. The aberrantly expressed lncRNAs in hepatocellular carcinoma may potentially prove useful as prognostic or diagnostic biomarkers [11-15]. Because of the important roles of MALAT1 in many diseases, especially cancer, MALAT1 is receiving more and more attention. It was confirmed that the expression of MALAT1 was upregulated in various types of tumors and that MALAT1 has a significant influence on tumor cell proliferation, migration, invasion, and apoptosis [1620]. The upregulated expression of MALAT1 was found in almost in every organ of the digestive system including hepatocellular carcinoma tissue, but its roles and the specific molecular mechanisms are still unclear.

In our study, we confirmed that MALAT1 was upregulated in hepatocellular carcinoma tissues compared with adjacent non-tumor tissues. We found that MALAT1 acted as a miRNA decoy for miR-142-3p and regulated the expression of miR-142-3p in hepatocellular carcinoma cells. The knockdown of MALAT1 inhibited proliferation, migration, invasion and EMT, and promoted cell apoptosis of hepatocellular carcinoma cells via miR-142-3p. MiR-142-3p suppressed cell proliferation migration, invasion and EMT, and promoted cell apoptosis by targeting SMAD5 in hepatocellular carcinoma. In the established xenograft model, MALAT1 promoted tumor growth by regulating the expression of miR-142-3p. Taken together, our results suggest that MALAT1 promoted cell proliferation, migration, and invasion by acting as a miRNA decoy for miR-142-3p.

\section{Methods \\ Cell lines}

The cell lines Bel-7402, Huh-7, HepG2 and SMMC7721 were obtained from the American Type Cell Culture (Manassas, VA). Human liver cell line HL-7702 was bought from the Shanghai Institute for Biological Sciences, Chinese Academy of Sciences. HL-7702 and Bel-7402 cells were cultured in RPMI-1640 containing $1 \%$ penicillin and streptomycin. The Huh-7, HepG2 and SMMC7721 cell lines were cultured in Dulbecco's Modified Eagle Media (DMEM). Both the RPMI-1640 and DMEM medium were supplemented with $10 \%$ fetal bovine serum (FBS) and cultured in a humidified incubator under $5 \% \mathrm{CO}_{2}$ at $37^{\circ} \mathrm{C}$.

\section{Clinical samples}

The hepatocellular carcinoma tissues and adjacent nontumor tissues were collected from hepatocellular carcinoma patients during surgery at Nanfang Hospital Affiliated to Southern Medical University. Informed consent was obtained from all patients and the study protocol was approved by the institutional ethics committee of Nanfang Hospital Affiliated to Southern Medical University. The tumor tissues were immediately frozen and kept at $-80{ }^{\circ} \mathrm{C}$.

\section{RNA isolation and quantitative real-time PCR (Q-PCR)}

Trizol reagent (Invitrogen, Carlsbad, CA) was used to extract the total RNA from hepatocellular carcinoma tissues and cells. Random primers, $1 \mu \mathrm{g}$ RNA template and Primescript reverse transcriptase (Takara, Japan) were used for single strand cDNA synthesis. Q-PCR was performed for MALAT1, glyceraldehyde 3-phosphate dehydrogenase (GAPDH), SMAD5, miR-142-3p and U6. Primer sequences were: MALAT1, Forward, 5'-ACG ATGGTGTCGAGGTCTTT- $3^{\prime}$ and reverse, $5^{\prime}$-TCCCAC CCAGCATTACAGTT-3'; GAPDH, Forward, 5'-TGT TCGTCATGGGTGTGAAC- $3^{\prime}$ and reverse, $5^{\prime}$-ATGGCA TGGACTGTGGTCAT-3'; SMAD5, Forward, 5' - CCA GCAGTAAAGCGATTGTTGG- $3^{\prime}$ and reverse, $5^{\prime}$-GGG GTAAGCCTTTTCTGTGAG-3'; miR-142-3p, Forward, 5'-ACACTCCAGCTGGGTGTAGTG TTTCCTACT TTA-3', and reverse, 5'-CTCAACTGGTGTCGTGGA '; U6, Forward, 5'-CTCGCTTCGGCAGCACA-3' and 
reverse, 5'-AACGCTTCACGAATTTGCGT-3'. The cDNA was amplified by an Applied Biosystems (ABI) step-one plus sequence detection system (Applied Biosystems, Foster City, CA). The relative expression was normalized to endogenous controls using the comparative cycle threshold (CT) method, and fold change was calculated as $2^{-\Delta \Delta \mathrm{Ct}}$ in gene expression.

\section{Cell viability assay}

For the cell viability assay, 3000 treated cells were seeded into 96-well microtiter plates. The medium was removed and fresh medium was added to each well, after the indicated treatment. After adding $10 \mu \mathrm{L}$ of CCK- 8 solution (CCK-8, Dojin, Japan) into each well, the cells were incubated for $2 \mathrm{~h}$ at $37^{\circ} \mathrm{C}$. The absorbance was measured at $450 \mathrm{~nm}$.

\section{Apoptosis assay}

Annexin V-fluorescein isothiocyanate/propidium iodid (Annexin V-FITC/PI) staining was used for the cell apoptosis assay. After treatment, the cells were collected and double stained with Annexin V-FITC/PI according to the manufacturer's instructions. FACS Calibur flow cytometer (Becton-Dickinson, Franklin Lakes, NJ) was used to analyzed the percentage of apoptotic cells.

\section{Cell migration assay}

A total of $1 \times 10^{5}$ cells/well was added into the upper chamber with $650 \mu \mathrm{L}$ medium containing $10 \% \mathrm{FBS}$ in the lower chamber. After $36 \mathrm{~h}$, the nonmigrated cells were removed using a cotton swab and the migrated cells were fixed by $4 \%$ formaldehyde, stained with $0.1 \%$ crystal violet. The field of view was randomly selected, and the total number of cells was counted.

\section{Matrigel invasion assay}

Matrigel-coated Transwell chambers (BD Biosciences, San Jose, CA) were used for the matrigel invasion assay according to the manufacturer's instructions. Briefly, 10,000 cells were seeded into the upper matrigel-coated chambers with DMEM containing 10\% FBS in the lower chamber. After $36 \mathrm{~h}$, non-invading cells in the upper chamber were removed by scrubbing with a cottontipped swab. The invaded cells were fixed with $4 \%$ paraformaldehyde and stained with $0.2 \%$ crystal violet. The total number of cells in a randomly selected field of view was counted. Six fields from each chamber were photographed.

\section{Western blot analysis}

Cells were homogenized with RIPA-containing buffer [50 mM Tris-Cl pH8.0, $150 \mathrm{mM} \mathrm{NaCl}, 0.02 \% \mathrm{NaN}_{3}, 0.1 \%$ SDS, $100 \mu \mathrm{g} / \mathrm{ml}$ phenylmethylsufonyl fluoride (PMSF),
$1 \mu \mathrm{g} / \mathrm{ml}$ aprotinin, $1 \%$ Triton]. After centrifugation, cell lysates $(100 \mu \mathrm{g} / \mathrm{lane})$ were subjected to $12 \%$ SDS-PAGE and transferred onto polyvinylidene difluoride membranes (Millipore). Antibodies against vimentin (Santa Cruz, 1:1000), GAPDH (CST, 1:1000), E-cadherin (CST, 1:1500) and HRP-conjugated goat anti-rabbit secondary antibodies (Promab, 1:1000) were used. Protein bands were detected by the enhanced chemiluminescence reaction and blot film was scanned.

\section{Cell transfection}

siRNA oligonucleotides and negative control were designed from the $\sim 350$ base pair highly conserved gVCIn4 region within the MALAT1 locus target sequence and synthesized by RiboBio (Co Ltd, China). The miR142-3p mimic, or control (scrambled negative controls) was designed and synthesized by RiboBio (Co Ltd, China). The SMAD5 full-length sequence was synthesized and subcloned into a pCDNA3.0 vector (Invitrogen, Shanghai, China). Lipofectamine 3000 (Invitrogen) was used for transfection of siRNA, miR-142-3p mimic, mimic control or plasmids into cells following to the manufacturer's protocol.

\section{Luciferase reporter assay}

Luciferase reporter plasmids (MALAT1-Wt and MALAT1-mut) were designed and constructed by Generay (Shanghai, China). Two luciferase reporters containing wild-type MALAT1 (psiCHECK2-MALAT1-WT, which encompassed the binding sites for miR-142-3p) or mutant MALAT1 (psiCHECK2- MALAT1-MUT, which encompassed the mutant sequence of the binding sites for miR-142-3p) were constructed. In order to analyze the interaction between MALAT1 and miR142-3p, the HEK293T cells were co-transfected with luciferase reporter plasmids and miRNA mimics by Lipofectamine $^{\mathrm{TM}} 3000$ transfection. Luciferase reporter plasmids (psiCHECK2-SMAD5-WT and psiCHECK2SMAD5-MUT) were also designed and constructed by Generay (Shanghai, China). In order to assess the interaction between SMAD5 and miR-142-3p, HEK293T cells were co-transfected with luciferase reporter plasmids and miRNA mimics by Lipofectamine ${ }^{\mathrm{TM}} 3000$ transfection. The dual-luciferase reporter assay system was used for luciferase activity analysis. The relative luciferase activity was calculated by the ratio of firefly luciferase activity to renilla luciferase activity.

\section{Animal studies}

For the xenograft tumor model, the stably over-expressing MALAT1 HepG2 cells and stably knocked down cell line were established by Lv-UBE2CP3, Lv-shMALAT1 and Lv-control viruses. A total of $4 \times 10^{6} \mathrm{HepG} 2$ cells 
were suspended in ice-cold PBS and injected in each nonobese diabetic/severe combined immunodeficiency (NOD/SCID) mouse (5 weeks old, male) to establish xenografts. The formula $V=a \times b^{2} / 2$ was used to calculated the tumor volume $(\mathrm{a}=$ long axis, $\mathrm{b}=$ short axis. All animal experimentation was approved by the Animal Care Committee of Nanfang Hospital, which is affiliated with Southern Medical University.

\section{EdU assay}

The EdU assay was performed as previously described [21]. Briefly, cells were treated with 5-ethynyl-2-deoxyuridine (Click-iT ${ }^{\circledR}$ Plus EdU Alexa- $647{ }^{\circledR}$ Imaging Kit, Life Technologies). Then, cells were washed with phosphatebuffered saline (PBS) and fixed by paraformaldehyde. For image capture, the FV10i confocal microscope (OLYMPUS, Japan) was used, and the EdU + cells in each field were counted.

\section{Immunofluorescence assays}

Immunofluorescence assays were performed as described in our previous study [22]. Antibodies against vimentin (Santa Cruz, 1:500) and E-cadherin (CST, 1:1500) were used and the images were captured using a FV10i confocal microscope (OLYMPUS, Japan).

\section{Immunocytochemistry}

Immunocytochemistry was performed according to the previously described method. Briefly, $4-\mu \mathrm{m}$ thick paraffin sections were deparaffinized and incubated in 3\% hydrogen peroxide to block endogenous peroxidase activity. The slides were blocked from nonspecific antibody binding and incubated with primary antibody against E-cadherin (1:200), N-cadherin (1:200), Ki-67 (1:300) and SMAD5 (1:300). All of the antibodies were purchased by Cell Signaling Technology.

\section{Statistical analysis}

All results are presented as the mean \pm standard error of the mean (SEM) from at least three independent experiments. Student's $t$ test was used to assess differences between two groups, and one-way analysis of variance was used for multiple comparisons. A value of $\mathrm{P}<0.05$ was considered statistically significant.

\section{Results}

\section{Overexpressed MALAT1 might act as a competing} endogenous RNA sponge for miR-142-3p in hepatocellular carcinoma

Firstly, we assessed the relative expression level of MALAT1 in hepatocellular carcinoma tissues and adjacent non-tumor tissues. As shown in Fig. 1a, the expression of MALAT1 was upregulated in hepatocellular carcinoma tissues. The hepatocellular carcinoma tissues were divided into two subsets: lymph node metastase positive and lymph node metastase negative. The level of MALAT1 in hepatocellular carcinoma tissues was significantly higher in lymph node metastase positive subsets than in lymph node metastase negative subsets (Fig. 1b). As shown in Fig. 1c, MALAT1 was significantly overexpressed in cancer subsets (Stage III and Stage IV) with respect to other subsets (Stage I and Stage II). By using the bioinformatics databases (Starbase, RNAhybrid) that predict potential lncRNA-miRNA interactions, we found that miR-142-3p was a putative MALAT1 binding miRNAs (Fig. 1d). Then, we analyzed the expression levels of miR-142-3p in hepatocellular carcinoma tissues and adjacent non-tumor tissues. The results showed that miR142-3p expression was downregulated in hepatocellular carcinoma tissues compared with adjacent non-tumor tissues (Fig. 1e). Further analysis of hepatocellular carcinoma specimens demonstrated that MALAT1 expression was negatively correlated with the expression of miR142-3p in corresponding specimens (Fig. 1f, $\mathrm{P}=0.0004$, $\mathrm{R}^{2}=0.3652$ ). Then, we measured the expression levels of MALAT1 and miR-142-3p in hepatocellular carcinoma cell lines and a human liver cell line. Notably, all the hepatocellular carcinoma cell lines-especially the two lines (HepG2, SMMC-7721)-had a higher level of MALAT1 than the human liver cell line. However, all of the hepatocellular carcinoma cell lines had a lower level of miR-142-3p than the human liver cell line (Fig. 1g). Next, the HepG2 and SMMC-7721 cell lines were selected for further study to assess the potential functional role of MALAT1. In HepG2 cells, the MALAT1 was overexpressed and we found that the level of miR142-3p was downregulated by MALAT1 overexpression (Fig. 1h). Luciferase activity assay was performed to verify the putative-binding sites between MALAT1 and miR-142-3p. The results showed that miR-142-3p downregulated the activity of luciferase reporter harboring wild-type MALAT1 but not the mutant MALAT1 (Fig. 1i). Collective data indicated that MALAT1 might act as a miRNA decoy for miR-142-3p and regulated the expression of miR-142-3p in hepatocellular carcinoma cells.

\section{Knockdown of MALAT1 inhibited the proliferation and promoted cell apoptosis of hepatocellular carcinoma cells via miR-142-3p}

Next, we explored the potential role of MALAT1 in hepatocellular carcinoma cells. The expression of MALAT1 was knocked down by siRNA. As shown in Fig. 2a, the expression of miR-142-3p in hepatocellular carcinoma cells was significantly increased by MALAT1 knockdown (Fig. 2a). The expression of MALAT1 and miR-142-3p 


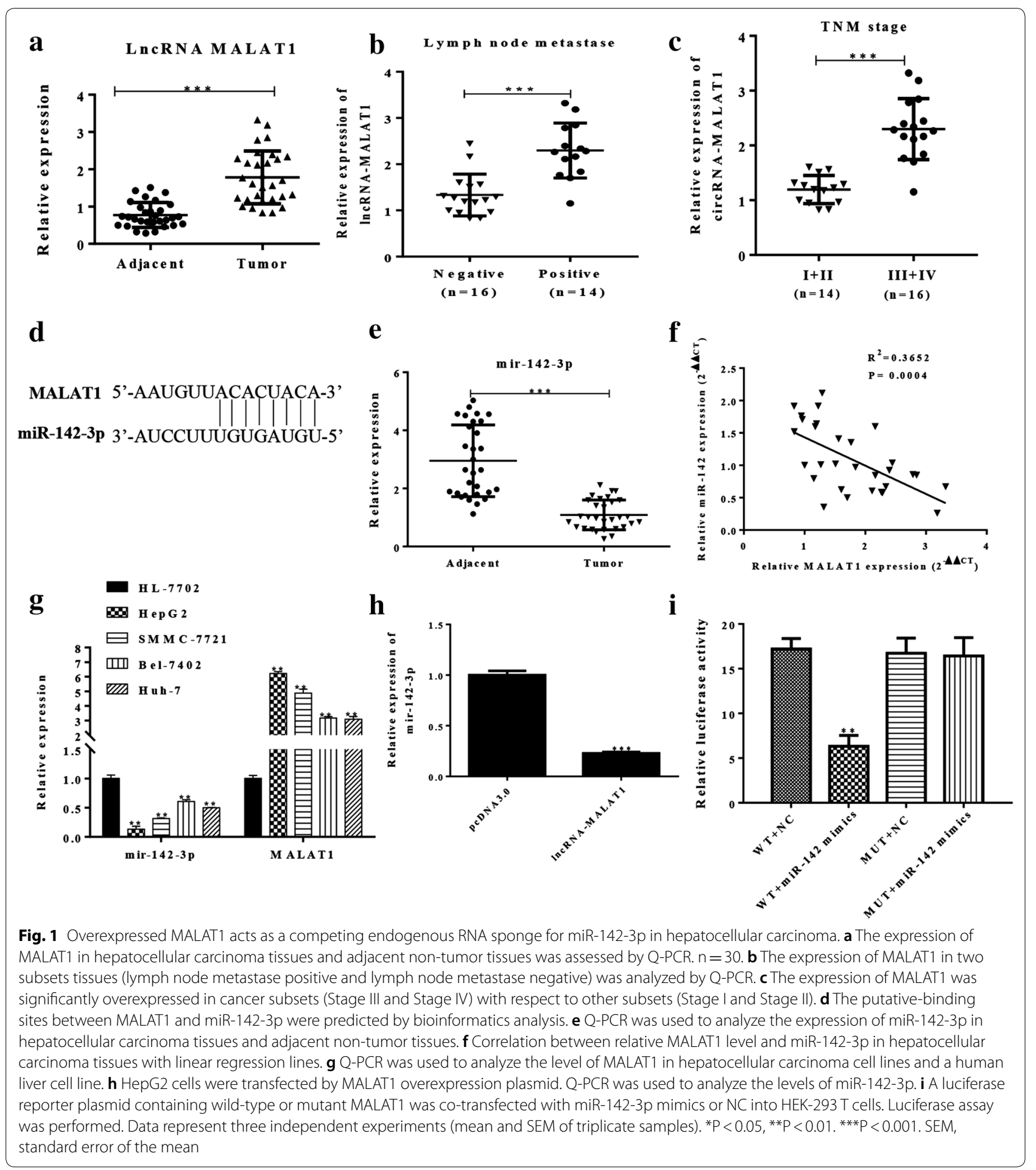

in HepG2 and SMMC-7721 cells was knocked down alone or together, and cell viability was assessed. As shown in Fig. 2b, c, the knockdown of MALAT1 significantly decreased the cell viability of HepG2 and SMMC-7721 cells, and co-transfection with miR-142-3p inhibitor reversed the effects of knockdown of MALAT1. MALAT1 had a similar effects on cell proliferation as assessed by EdU assay (Fig. 2d). For cell apoptosis, the knockdown of MALAT1 significantly promoted the cell apoptosis of HepG2 and SMMC-7721 cells, while 


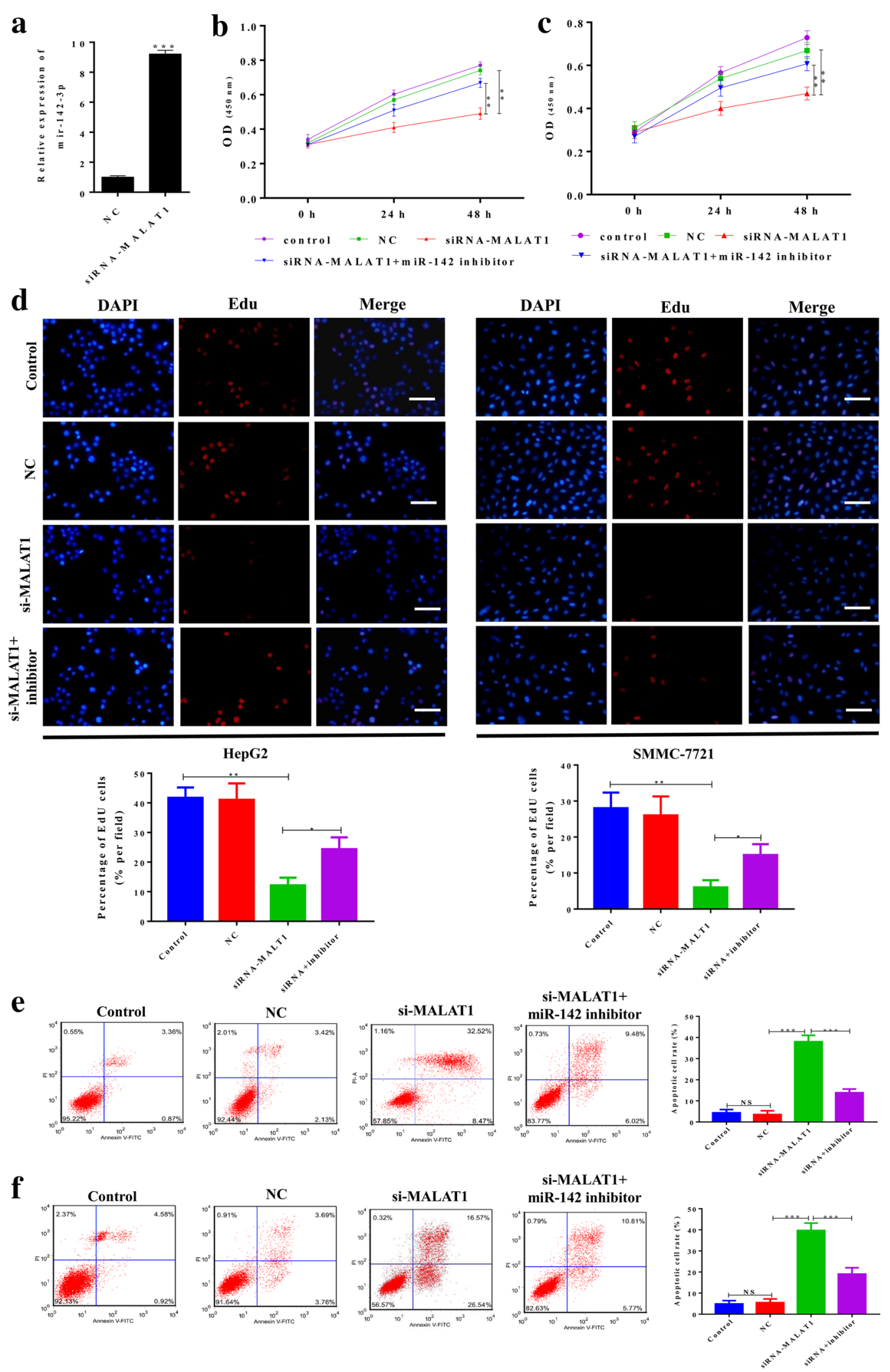


(See figure on previous page.)

Fig. 2 Knockdown of MALAT1 inhibited the proliferation and promoted cell apoptosis of hepatocellular carcinoma cells via miR-142-3p. The expression of MALAT1 in HepG2 and SMMC-7721 cells was knocked down by transfecting with MALAT1 siRNAs or si-control. NC was used as the control. The miR-142-3p inhibitor was used to knock down the level of miR-142-3p. a Q-PCR was used to analyze the level of miR-142-3p in HepG2 cells after transfection with MALAT1 siRNAs or control siRNAs (NC). b, c Cell viability was assessed by CCK-8 assay after the indicated transfection in HepG2 and SMMC-7721 cells. d The cell proliferation of HepG2 and SMMC-7721 cells after the indicated transfection was assessed by EdU assay. Representative images of the cells are shown. Scale bar, $100 \mu \mathrm{m}$. e, f Cell apoptosis of HepG2 and SMMC-7721 cells after the indicated transfection was evaluated by flow cytometry. Data represent three independent experiments (mean and SEM of triplicate samples). ${ }^{*} P<0.05,{ }^{* *} P<0.01$. ***P $<0.001$. SEM, standard error of the mean

co-transfection with miR-142-3p inhibitor reversed the effects of knockdown of MALAT1 on cell apoptosis (Fig. 2e, f).

\section{Knockdown of MALAT1 inhibited the migration and invasion of hepatocellular carcinoma cells by EMT}

Then, we further ascertained the role of MALAT1 in cell migration and invasion of hepatocellular carcinoma cells. A shown in Fig. 3a-c, the knockdown of MALAT1 significantly inhibited the migration and invasion potency of HepG2 and SMMC-7721 cells. Cotransfection with miR142-3p inhibitor reversed the effects of knockdown of MALAT1 on migration and invasion potency of HepG2 and SMMC-7721 cells. Analysis of EMT makers by Western blot showed that knockdown of MALAT1 decreased the protein level of vimentin and increased the protein level of E-cadherin in both HepG2 and SMMC-7721 cells. Co-transfection with miR-142-3p inhibitor reversed the effects of MALAT1 knockdown on protein levels of vimentin and E-cadherin (Fig. 3d). Similarly, immunofluorescence assay also confirmed the roles of MALAT1 in regulating the protein levels of vimentin and E-cadherin (Fig. 3e).

\section{MiR-142-3p suppressed cell proliferation and promoted cell apoptosis by targeting SMAD5 in hepatocellular carcinoma}

To further explore the molecular mechanism of miR$142-3 p$ in regulating cell proliferation, migration and invasion of hepatocellular carcinoma cells, we searched bioinformatics databases. SMAD5 was identified as a putative target of miR-142-3p (Fig. 4a). The expression of SMAD5 in hepatocellular was upregulated in carcinoma tissues compared with adjacent non-tumor tissues (Fig. 4b). The mRNA level of SMAD5 in HepG2 and SMMC-7721 cells was higher compared with three other cell lines (Fig. 4c). In HepG2 and SMMC-7721 cells, miR-142-3p was ectopically expressed by transfection of miR-142-3p mimic, and the transfection efficiency was confirmed (Fig. 4d). As shown in Fig. 4e, the mRNA level of SMAD5 in HepG2 and SMMC-7721 cells was significantly decreased by miR-142-3p ectopic expression. The protein level of SMAD5 in HepG2 and SMMC-7721 cells was also significantly decreased by miR-142-3p ectopic expression (Fig. 4f). Luciferase activity assays confirmed that SMAD5 was a target of miR-142-3p (Fig. 4g).

Next, we explored the roles of miR-142-3p and SMAD5 in HepG2 and SMMC-7721 cells. As shown in Fig. 4h, the ectopic expression of SMAD5 promoted the viability of HepG2 and SMMC-7721 cells, and co-transfection with miR-142-3p mimic reversed the effects of SMAD5 overexpression. Similar results were found for cell proliferation as assessed by EdU assay (Fig. 4i). The ectopic expression of SMAD5 significantly inhibited the cell apoptosis of HepG2 and SMMC-7721 cells, while the cotransfection with miR-142-3p mimic reversed the effects of SMAD5 overexpression in cell apoptosis (Fig. 4j).

\section{MiR-142-3p suppressed migration, invasion and EMT by targeting SMAD5 in hepatocellular carcinoma}

The role of MALAT1 in cell migration and invasion of hepatocellular carcinoma cells was further explored. As shown in Fig. 5a-c, the ectopic expression of SMAD5 significantly increased migration and invasion viability of HepG2 and SMMC-7721 cells. Co-transfection with miR-142-3p mimic reversed the effects of ectopic expression of SMAD5 on migration and invasion viability of HepG2 and SMMC-7721 cells. The ectopic expression of miR-142-3p inhibited the migration and invasion potency of HepG2 and SMMC-7721 cells (Fig. 5a-c). Regarding EMT, Western blot indicated that the overexpression of SMAD5 increased the protein level of vimentin and decreased the level of E-cadherin in both HepG2 and SMMC-7721 cells. Co-transfection with miR-142-3p mimic reversed the effects of ectopic expression of SMAD5 on protein levels of vimentin and E-cadherin (Fig. 5d). Similar results were found by immunofluorescence assay (Fig. 5e). Collectively, miR-142-3p suppressed migration, invasion, and EMT by targeting SMAD5 in hepatocellular carcinoma.

\section{MALAT1 promoted tumor growth by regulating the expression of miR-142-3p}

MALAT1 was stably knocked down and overexpressed in HepG2 cells to establish stable cell lines. Then, the cell lines were used to establish a xenograft model to 


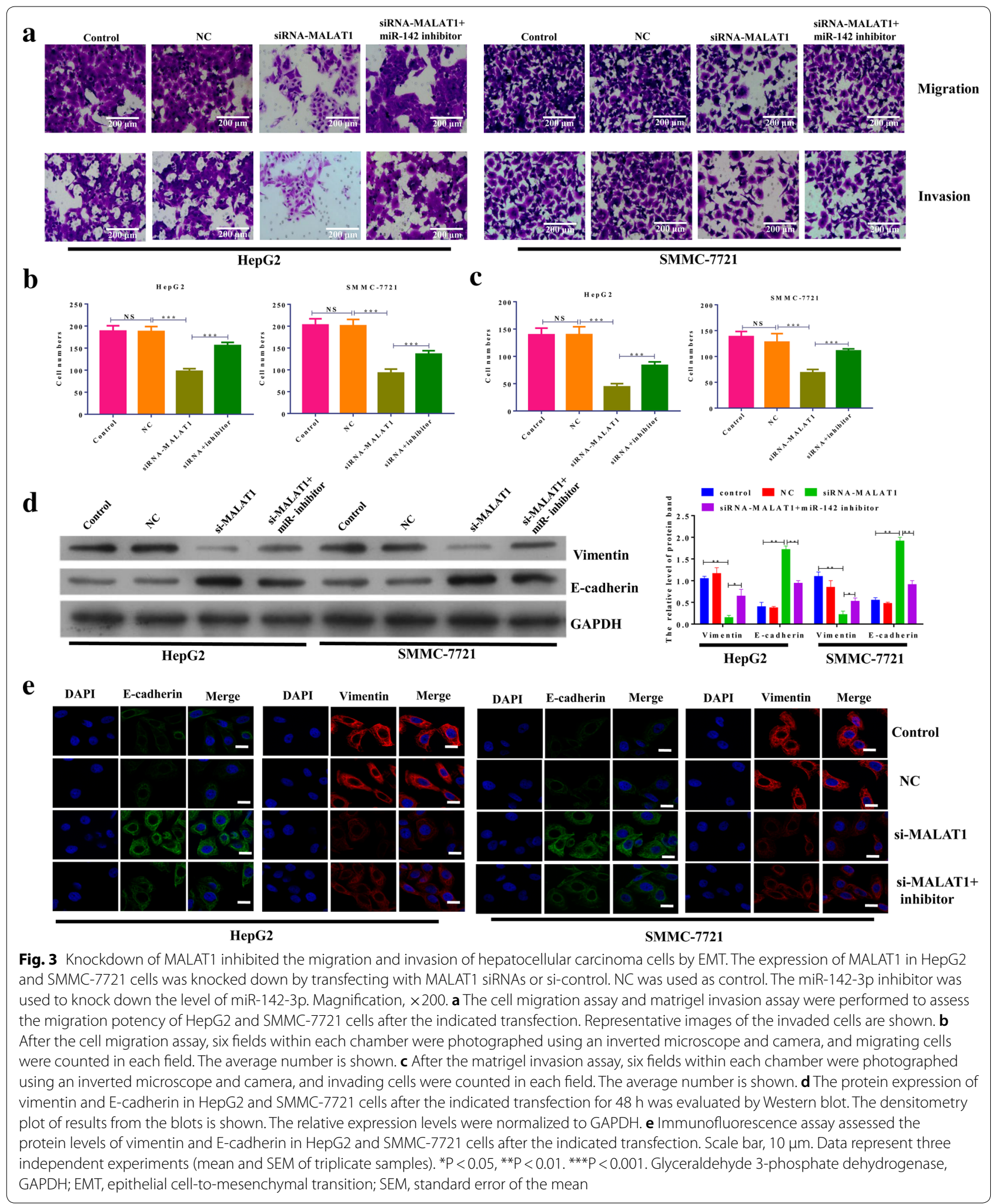




\section{a}
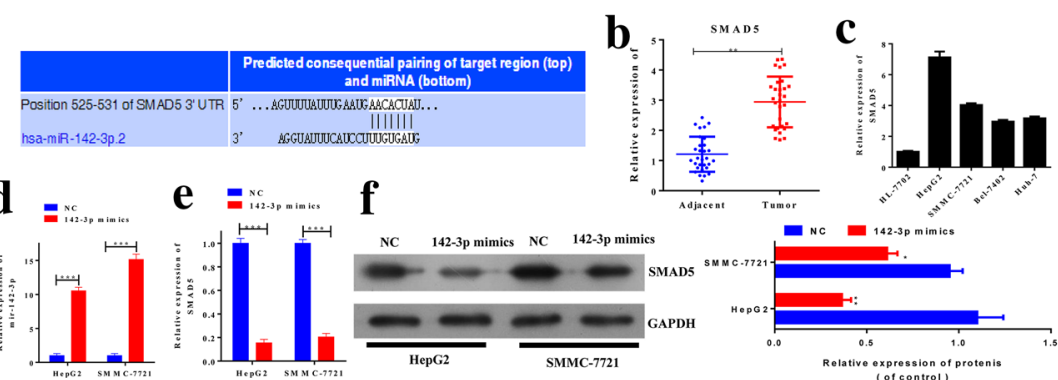

g

h
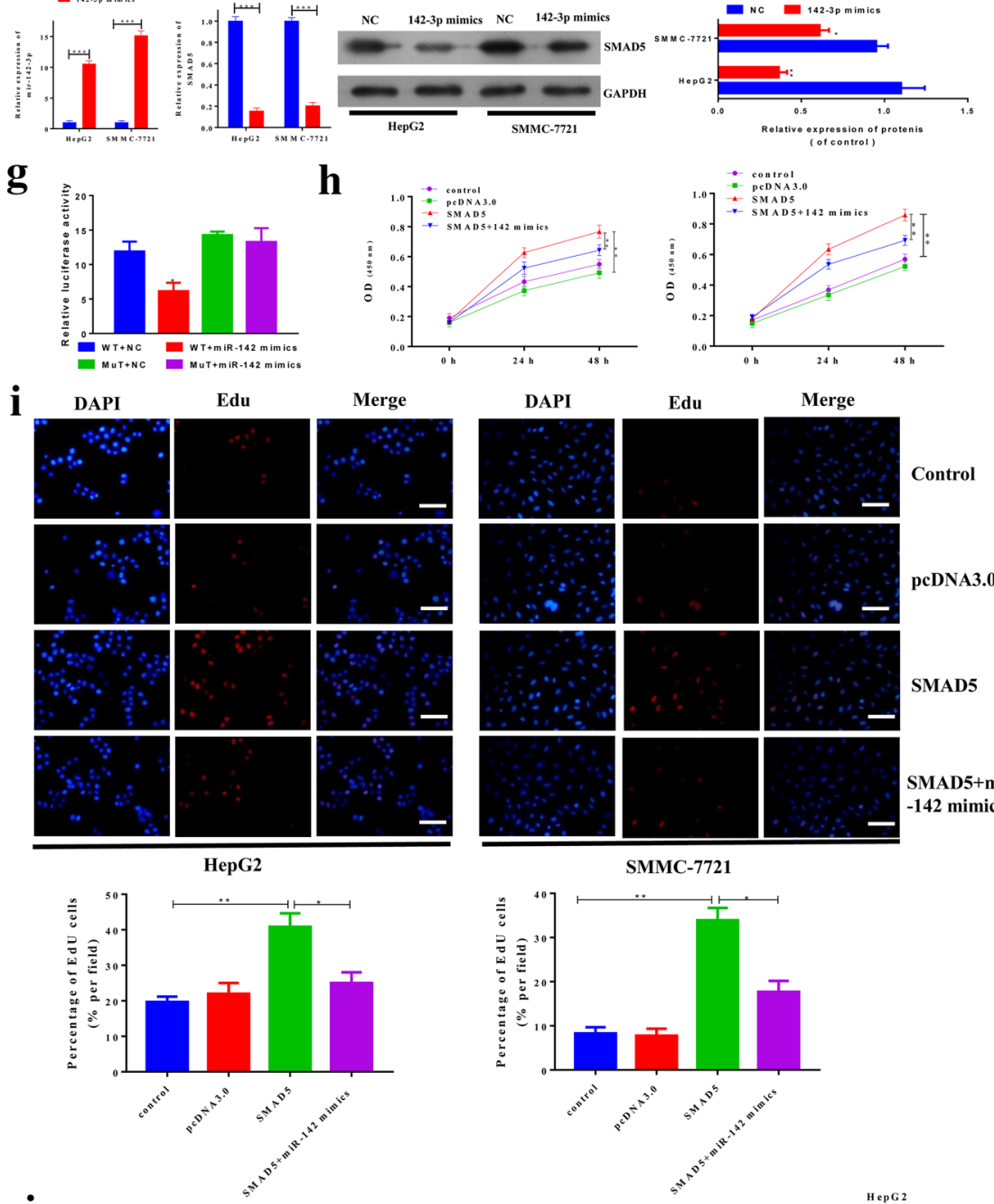

SMAD5
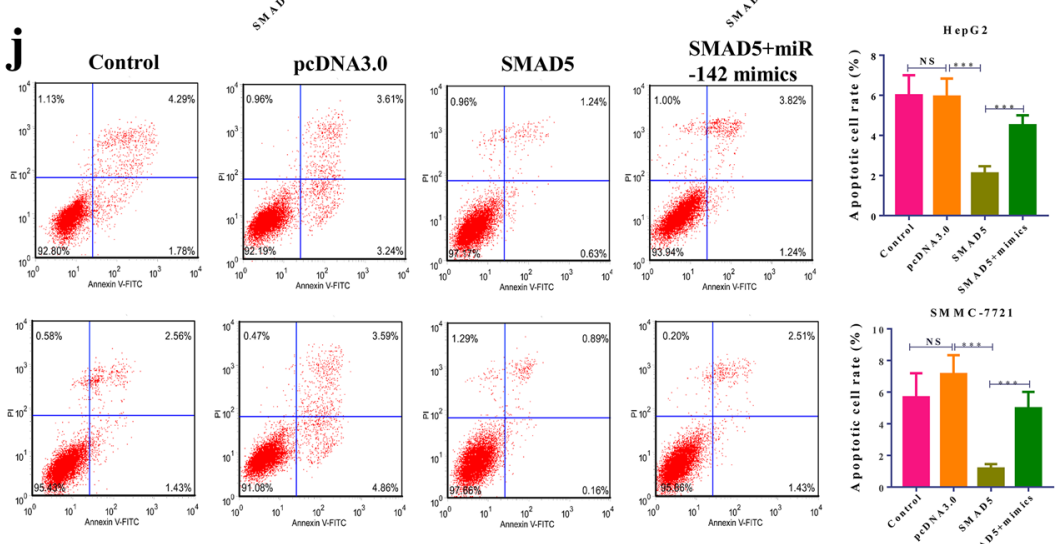
(See figure on previous page.)

Fig. 4 MiR-142-3p suppressed the cell proliferation and promoted cell apoptosis by targeting SMAD5 in hepatocellular carcinoma. a Schematic diagrams of the putative interactions between miR-142-3p and SMAD5. b The expression of SMAD5 in hepatocellular carcinoma tissues and adjacent non-tumor tissues was assessed by Q-PCR. c Q-PCR was used to analyze the mRNA level of SMAD5 in 5 hepatocellular carcinoma cell lines. d HepG2 cells were transfected by miR-142-3p mimic control mimic (NC), and the level of miR-142-3p was analyzed by Q-PCR. e The HepG2 and SMMC-7721 cells were transfected by miR-142-3p mimic control mimic (NC); the mRNA was analyzed by Q-PCR. $\mathbf{f} H e p G 2$ and SMMC-7721 cells were transfected by miR-142-3p mimic control mimic (NC). The protein level of SMAD5 was analyzed by Western blot. $\mathbf{g}$ The luciferase reporter plasmid containing wild-type or mutant SMAD5 was co-transfected with miR-142-3p mimics or control mimic into HEK-293 T cells. Luciferase activity was analyzed and normalized to Renilla activity. $\mathbf{h}$ SMAD5 in HepG2 and SMMC-7721 cells was ectopically expressed using a plasmid containing SMAD5. The plasmid vector pCDNA3.0 was used as the control. The miR-142-3p mimic control mimic (NC) was also used as indicated in the figure. Cell viability was assessed by CCK-8 assay after the indicated transfection in HepG2 and SMMC-7721 cells. i The cell proliferation of HepG2 and SMMC-7721 cells after the indicated transfection was assessed by EdU assay. Representative images of the cells are shown. Scale bar, 100 um. $\mathbf{j}$ Cell apoptosis of HepG2 and SMMC-7721 cells after the indicated transfection was assessed by flow cytometry. Data represent three independent experiments (mean and SEM of triplicate samples). ${ }^{*} \mathrm{P}<0.05$, ${ }^{* *} \mathrm{P}<0.01$. ${ }^{* * *} \mathrm{P}<0.001$. SEM, standard error of the mean

investigate the role of MALAT1 in tumor growth. As shown in Fig. 6a, b, the knockdown of MALAT1 significantly inhibited tumor growth, while the ectopic expression of MALAT1 significantly promoted tumor growth. The expression of H19 in tumor tissues of the xenograft models was confirmed (Fig. 6c). Furthermore, the expression of miR-142-3p and E-cadherin was significantly increased in the MALAT1 knockdown group compared with the control group. The expression of miR-142-3p and E-cadherin was significantly decreased in the ectopically expressed MALAT1 group compared with the control group. The MALAT1 knockdown group showed a decrease in expression while the ectopically expressed MALAT1 group showed an increase in expression of vimentin (Fig. 6c, d). In addition, knockdown of MALAT1 decreased the expression of Ki-67 and SMAD5 while ectopic expression of MALAT1 significantly increased the expression of Ki-67 and SMAD5 (Fig. 6e, f).

\section{Discussion}

Previously discovered and widely studied as a lncRNA, MALAT1 has been shown to be involved in the metastasis of early-stage non-small cell lung cancer (NSCLC) and is considered a prognostic marker for stage I of NSCLC [23]. In addition, MALAT1 is associated with other noncancer diseases, including myocardial infarction and hyperglycemia [18, 24-27]. In the cardiovascular system, MALAT1 was is involved in endothelial cell function and vessel growth [24, 25]. In addition, MALAT1 expression in tissues associated with myocardial infarction is upregulated and plays a vital role in cardiovascular disease [26]. MALAT1 is highly expressed in hepatocellular carcinoma cell lines when compared to normal liver cells [28], and MALAT1-miR-195-EGFR axis was found to play an important role in hepatocellular carcinoma [29]. Our results suggested that the MALAT1 promoted cell proliferation, migration and invasion by the MALAT1miR-142-3p-SMAD5 axis.
The current study supports findings from other studies that miR-142 is a key regulator of many biological processes and related signaling pathways in embryonic development, homeostasis and disease processes [30]. MiR-142 has been shown to be preferentially expressed in embryonic tissue, hematopoietic tissues, immune cells, tumors tissues, and many other kinds of tissues [31]. In addition, miR-142 has been shown to play important functions in disease, including functional role in cancer, lineage differentiation of hematopoietic cells, virus infection, inflammation, and immune tolerance [30, 32-34]. Regarding cervical cancer, the expression of miR-142-3p is reduced in cervical cancer epithelial cells compared with healthy cervical epithelial cells, suggesting a regulatory role [35, 36]. MicroRNA-142-3p inhibits cell proliferation and invasion of cervical cancer cells by targeting Frizzled class receptor 7 (FZD7). MiR-142-3p had tumor suppressive effects in cell proliferation and invasion in Hela and SiHa cells [36]. Similarly miR-142-3p is involved in tumor progression and invasion in hepatocellular carcinoma cells [37, 38].

In our study, we found that the expression of miR142-3p was downregulated in hepatocellular carcinoma tissues compared with adjacent non-tumor tissues. MALAT1 acts as a miRNA decoy for miR-142-3p and regulates the expression of miR-142-3p in hepatocellular carcinoma cells. In addition, miR-142-3p suppresses cell proliferation, migration, invasion and EMT, and promotes cell apoptosis by targeting SMAD5 in hepatocellular carcinoma. As an important biological process, EMT is characterized by a progressive loss of epithelial characteristics and acquisition of mesenchymal properties [39]. Collectively, studies have reported that activation of EMT reprogramming plays an important role in tumor progression and drug resistance [40]. In cancer epithelial cancer cells, the activation of EMT is accompanied by downregulated expression of adhesion molecules (E-cadherin) and upregulated expression of mesenchymal 


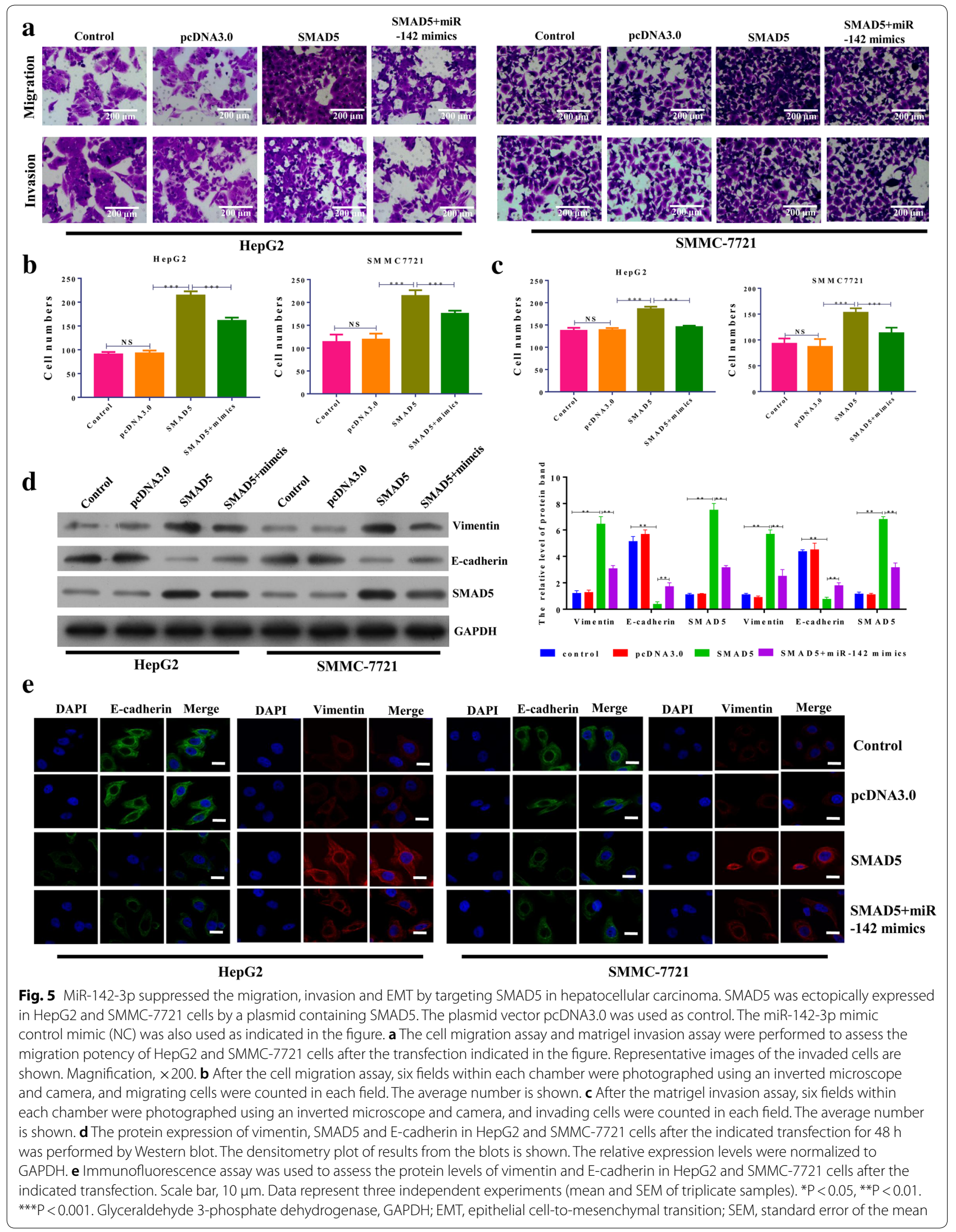




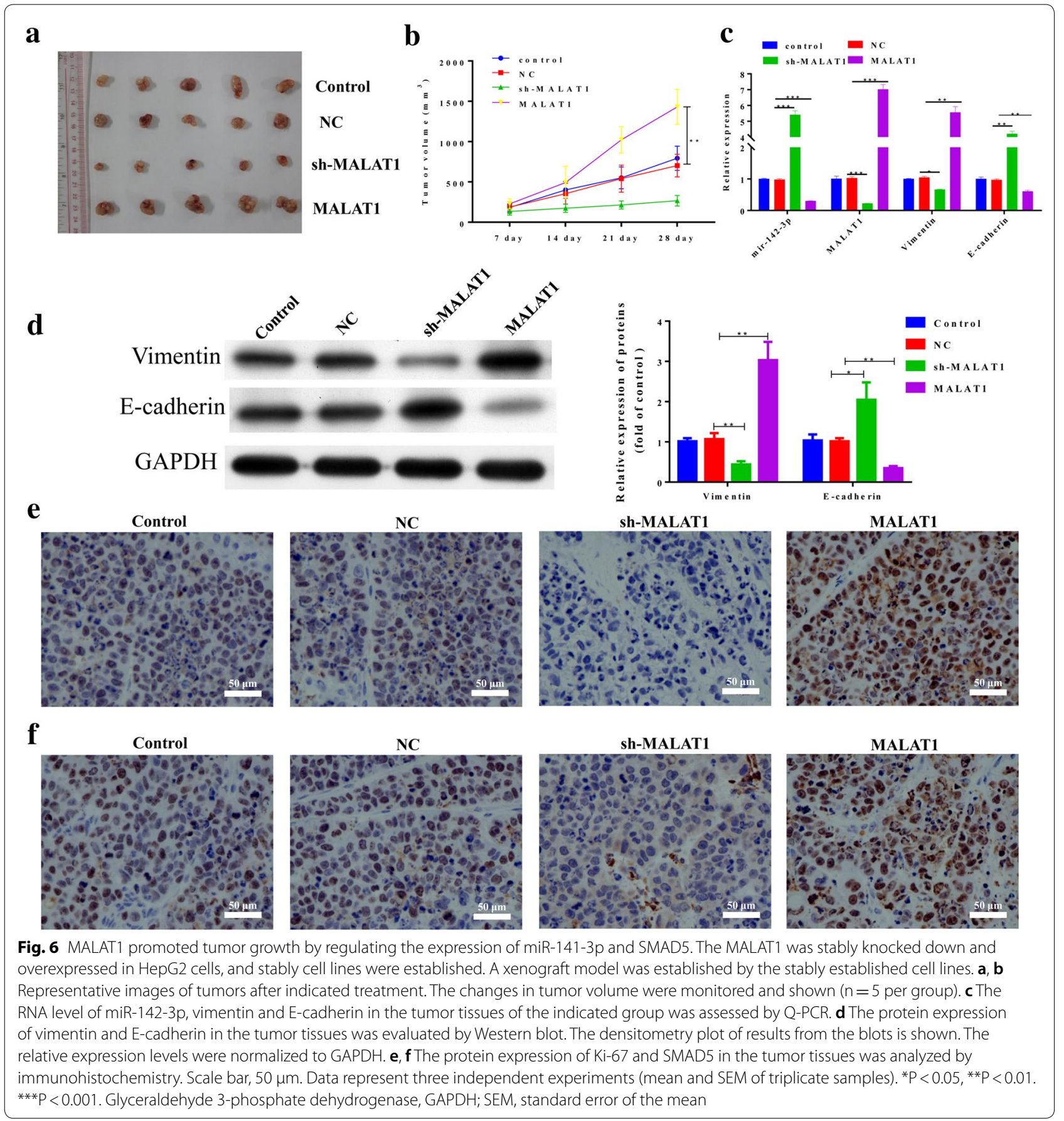

proteins (N-cadherin and vimentin) that favor cell motility and invasion [41-43]. Our study showed that the knockdown of MALAT1 inhibited the migration and invasion of hepatocellular carcinoma cells by suppressing EMT. The loss of EMT makers may lead to the inhibition of migration and invasion of hepatocellular carcinoma cells.
In summary, we have characterized the roles of MALAT1 in hepatocellular carcinoma and specifically delineated potential molecular mechanisms. The MALAT1-miR-142-3p-SMAD5 axis plays an important role in cell proliferation, migration, and invasion of hepatocellular carcinoma cells. The findings from the current study have furthered our understanding of hepatocellular 
carcinoma and identified potential targets for clinical treatment.

\section{Abbreviations}

MALAT1: metastasis-associated lung adenocarcinoma transcript 1; miR-142-3p: microRNA-142-3p; CCK-8: Cell Counting Kit-8; EMT: epithelial cell-to-mesenchymal transition; IncRNAs: long noncoding RNAs.

\section{Authors' contributions}

QFY, JYZ and DHY conceived and designed the research. QFY, LYX, ZJC, $X C L$ and JYZ carried out experiments and analyzed the data. QFY, HHO, JYZ and $\mathrm{DHY}$ wrote the main manuscript. All authors discussed the results and commented on the manuscript. All authors read and approved the final manuscript.

\section{Author details}

${ }^{1}$ Department of Hepatobiliary Surgery, The Second Hospital of Longyan, Fujian, China. ${ }^{2}$ Department of Hepatobiliary Surgery, Nanfang Hospital Affiliated to Southern Medical University, Guangzhou, China. ${ }^{3}$ The Second Affiliated Hospital of Shantou University Medical College, Shantou, China. ${ }^{4}$ Department of Hepatobiliary and Pancreatic Surgery, Zhongshan Hospital, Xiamen University, Xiamen, China.

\section{Acknowledgements}

Not applicable.

\section{Competing interests}

The authors declare that they have no competing interests.

\section{Availability of data and materials}

The datasets used and/or analysed during the current study are available from the corresponding author upon request.

\section{Consent for publication}

Not applicable.

\section{Ethics approval and consent to participate}

This present study was approved by the second hospital of Longyan Ethics Committee for Human Research.

\section{Funding}

This research was supported by The Xiamen Science and Technology Plan Project (3502Z20164020); Natural Science Foundation of Fujian Province (2018J01153, 2019J01551); The Project Funding for the Training of Young Talents in the Health System of Fujian Province (2014-ZQNJC-42). Science and Technology Planning Project of Guangdong Province (2013B022000069). National Natural Science Foundation of China (8187111677).

\section{Publisher's Note}

Springer Nature remains neutral with regard to jurisdictional claims in published maps and institutional affiliations.

Received: 26 January 2019 Accepted: 22 April 2019

Published online: 10 May 2019

\section{References}

1. Ferlay J, Soerjomataram I, Dikshit R, Eser S, Mathers C, Rebelo M, Parkin DM, Forman D, Bray F. Cancer incidence and mortality worldwide: sources, methods and major patterns in GLOBOCAN 2012. Int J Cancer. 2015;136:E359-86.

2. Liu J, Yang HI, Lee MH, Lu SN, Jen CL, Batrla-Utermann R, Wang LY, You SL, Hsiao CK, Chen PJ, Chen CJ, R.E.V.E.A.L.H.S. Group. Spontaneous seroclearance of hepatitis B seromarkers and subsequent risk of hepatocellular carcinoma. Gut. 2014;63:1648-57.

3. Tang LSY, Covert E, Wilson E, Kottilil S. Chronic hepatitis b infection: a review. JAMA. 2018;319:1802-13.
4. Llovet JM, Zucman-Rossi J, Pikarsky E, Sangro B, Schwartz M, Sherman M, Gores G. Hepatocellular carcinoma. Nat Rev. 2016;2:16018.

5. Nault JC, Galle PR, Marquardt JU. The role of molecular enrichment on future therapies in hepatocellular carcinoma. J Hepatol. 2018;69:237-47.

6. Llovet JM, Montal R, Sia D, Finn RS. Molecular therapies and precision medicine for hepatocellular carcinoma. Clin Oncol. 2018;30:1.

7. Zhou H, Xu Q, Ni C, Ye S, Xu X, Hu X, Jiang J, Hong Y, Huang D, Yang L. Prospects of noncoding RNAs in hepatocellular carcinoma. Biomed Res Int. 2018:2018:6579436.

8. Prensner JR, Chinnaiyan AM. The emergence of IncRNAs in cancer biology. Cancer Discov. 2011;1:391-407.

9. Yang F, Zhang L, Huo XS, Yuan JH, Xu D, Yuan SX, Zhu N, Zhou WP, Yang GS, Wang YZ, Shang JL, Gao CF, Zhang FR, Wang F, Sun SH. Long noncoding RNA high expression in hepatocellular carcinoma facilitates tumor growth through enhancer of zeste homolog 2 in humans. Hepatology. 2011;54:1679-89.

10. Schmitt AM, Chang HY. Long noncoding RNAs in cancer pathways. Cancer Cell. 2016:29:452-63.

11. Huang JF, Guo YJ, Zhao CX, Yuan SX, Wang Y, Tang GN, Zhou WP, Sun SH. Hepatitis B virus $X$ protein ( $\mathrm{HBX}$ )-related long noncoding RNA (IncRNA) down-regulated expression by $\mathrm{HBx}$ (Dreh) inhibits hepatocellular carcinoma metastasis by targeting the intermediate filament protein vimentin. Hepatology. 2013;57:1882-92.

12. Yuan JH, Yang F, Wang F, Ma JZ, Guo YJ, Tao QF, Liu F, Pan W, Wang TT, Zhou CC, Wang SB, Wang YZ, Yang Y, Yang N, Zhou WP, Yang GS, Sun $\mathrm{SH}$. A long noncoding RNA activated by TGF-beta promotes the invasion-metastasis cascade in hepatocellular carcinoma. Cancer Cell. 2014;25:666-81.

13. Gupta RA, Shah N, Wang KC, Kim J, Horlings HM, Wong DJ, Tsai MC, Hung T, Argani P, Rinn JL, Wang Y, Brzoska P, Kong B, Li R, West RB, van de Vijver MJ, Sukumar S, Chang HY. Long non-coding RNA HOTAIR reprograms chromatin state to promote cancer metastasis. Nature. 2010;464:1071-6.

14. Prensner JR, Iyer MK, Sahu A, Asangani IA, Cao Q, Patel L, Vergara IA, Davicioni E, Erho N, Ghadessi M, Jenkins RB, Triche TJ, Malik R, Bedenis R, McGregor N, Ma T, Chen W, Han S, Jing X, Cao X, Wang X, Chandler B, Yan W, Siddiqui J, Kunju LP, Dhanasekaran SM, Pienta KJ, Feng FY, Chinnaiyan AM. The long noncoding RNA SChLAP1 promotes aggressive prostate cancer and antagonizes the SWI/SNF complex. Nat Genet. 2013;45:1392-8.

15. Hung CL, Wang LY, Yu YL, Chen HW, Srivastava S, Petrovics G, Kung HJ. A long noncoding RNA connects c-Myc to tumor metabolism. Proc Natl Acad Sci USA. 2014:111:18697-702.

16. Djebali S, Davis CA, Merkel A, Dobin A, Lassmann T, Mortazavi A, Tanzer A, Lagarde J, Lin W, Schlesinger F, Xue C, Marinov GK, Khatun J, Williams BA, Zaleski C, Rozowsky J, Roder M, Kokocinski F, Abdelhamid RF, Alioto T, Antoshechkin I, Baer MT, Bar NS, Batut P, Bell K, Bell I, Chakrabortty S, Chen X, Chrast J, Curado J, Derrien T, Drenkow J, Dumais E, Dumais J, Duttagupta R, Falconnet E, Fastuca M, Fejes-Toth K, Ferreira P, Foissac S, Fullwood MJ, Gao H, Gonzalez D, Gordon A, Gunawardena H, Howald C, Jha S, Johnson R, Kapranov P, King B, Kingswood C, Luo OJ, Park E, Persaud K, Preall JB, Ribeca P, Risk B, Robyr D, Sammeth M, Schaffer L, See LH, Shahab A, Skancke J, Suzuki AM, Takahashi H, Tilgner H, Trout D, Walters N, Wang H, Wrobel J, Yu Y, Ruan X, Hayashizaki Y, Harrow J, Gerstein M, Hubbard T, Reymond A, Antonarakis SE, Hannon G, Giddings MC, Ruan Y, Wold B, Carninci P, Guigo R, Gingeras TR. Landscape of transcription in human cells. Nature. 2012;489:101-8.

17. Ponting CP, Oliver PL, Reik W. Evolution and functions of long noncoding RNAs. Cell. 2009;136:629-41.

18. Liu JY, Yao J, Li XM, Song YC, Wang XQ, Li YJ, Yan B, Jiang Q. Pathogenic role of IncRNA-MALAT1 in endothelial cell dysfunction in diabetes mellitus. Cell Death Dis. 2014;5:e1506.

19. Hu L, Wu Y, Tan D, Meng H, Wang K, Bai Y, Yang K. Up-regulation of long noncoding RNA MALAT1 contributes to proliferation and metastasis in esophageal squamous cell carcinoma. J Exp Clin Cancer Res. 2015;34:7.

20. Okugawa Y, Toiyama Y, Hur K, Toden S, Saigusa S, Tanaka K, Inoue Y, Mohri Y, Kusunoki M, Boland CR, Goel A. Metastasis-associated long non-coding RNA drives gastric cancer development and promotes peritoneal metastasis. Carcinogenesis. 2014;35:2731-9.

21. Zellmer VR, Schnepp PM, Fracci SL, Tan X, Howe EN, Zhang S. Tumorinduced stromal STAT1 accelerates breast cancer via deregulating tissue homeostasis. MCR. 2017;15:585-97. 
22. Lu L, Sun K, Chen X, Zhao Y, Wang L, Zhou L, Sun H, Wang H. Genomewide survey by ChIP-seg reveals $Y Y 1$ regulation of lincRNAs in skeletal myogenesis. EMBO J. 2013;32:2575-88.

23. Ji P, Diederichs S, Wang W, Boing S, Metzger R, Schneider PM, Tidow N, Brandt B, Buerger H, Bulk E, Thomas M, Berdel WE, Serve H, Muller-Tidow C. MALAT-1, a novel noncoding RNA, and thymosin beta4 predict metastasis and survival in early-stage non-small cell lung cancer. Oncogene. 2003;22:8031-41.

24. Michalik KM, You X, Manavski Y, Doddaballapur A, Zornig M, Braun T, John D, Ponomareva Y, Chen W, Uchida S, Boon RA, Dimmeler S. Long noncoding RNA MALAT1 regulates endothelial cell function and vessel growth. Circ Res. 2014;114:1389-97.

25. Uchida S, Dimmeler S. Long noncoding RNAs in cardiovascular diseases. Circ Res. 2015;116:737-50.

26. Vausort M, Wagner DR, Devaux Y. Long noncoding RNAs in patients with acute myocardial infarction. Circ Res. 2014;115:668-77.

27. Puthanveetil P, Chen S, Feng B, Gautam A, Chakrabarti S. Long noncoding RNA MALAT1 regulates hyperglycaemia induced inflammatory process in the endothelial cells. J Cell Mol Med. 2015;19:1418-25.

28. Lai MC, Yang Z, Zhou L, Zhu QQ, Xie HY, Zhang F, Wu LM, Chen LM, Zheng SS. Long non-coding RNA MALAT-1 overexpression predicts tumor recurrence of hepatocellular carcinoma after liver transplantation. Med Oncol. 2012;29:1810-6

29. Liu D, Zhu Y, Pang J, Weng X, Feng X, Guo Y. Knockdown of long noncoding RNA MALAT1 inhibits growth and motility of human hepatoma cells via modulation of miR-195. J Cell Biochem. 2018;119:1368-80.

30. Shrestha A, Mukhametshina RT, Taghizadeh S, Vasquez-Pacheco E, Cabrera-Fuentes H, Rizvanov A, Mari B, Carraro G, Bellusci S. MicroRNA-142 is a multifaceted regulator in organogenesis, homeostasis, and disease. Dev Dyn. 2017;246:285-90.

31. Chen ZY, Chen F, Cao N, Zhou ZW, Yang HT. miR-142-3p contributes to early cardiac fate decision of embryonic stem cells. Stem Cells Int. 2017;2017:1769298.

32. Carraro G, Shrestha A, Rostkovius J, Contreras A, Chao CM, El Agha E, Mackenzie B, Dilai S, Guidolin D, Taketo MM, Gunther A, Kumar ME, Seeger W, De Langhe S, Barreto G, Bellusci S. miR-142-3p balances proliferation and differentiation of mesenchymal cells during lung development. Development. 2014:141:1272-81.
33. Chakkalakal JV, Jones KM, Basson MA, Brack AS. The aged niche disrupts muscle stem cell quiescence. Nature. 2012;490:355-60.

34. Chen CZ, Schaffert S, Fragoso R, Loh C. Regulation of immune responses and tolerance: the microRNA perspective. Immunol Rev. 2013:253:112-28

35. Xia C, Liang S, He Z, Zhu X, Chen R, Chen J. Metformin, a first-line drug for type 2 diabetes mellitus, disrupts the MALAT1/miR-142-3p sponge to decrease invasion and migration in cervical cancer cells. Eur J Pharmacol. 2018;830:59-67.

36. Deng B, Zhang Y, Zhang S, Wen F, Miao Y, Guo K. MicroRNA-142-3p inhibits cell proliferation and invasion of cervical cancer cells by targeting FZD7. Tumour Biol. 2015;36:8065-73.

37. He C, Liu Z, Jin L, Zhang F, Peng X, Xiao Y, Wang X, Lyu Q, Cai X. IncRNA TUG1-mediated Mir-142-3p downregulation contributes to metastasis and the epithelial-to-mesenchymal transition of hepatocellular carcinoma by targeting ZEB1. Cell Physiol Biochem. 2018;48:1928-41.

38. Hua S, Liu C, Liu L, Wu D. miR-142-3p inhibits aerobic glycolysis and cell proliferation in hepatocellular carcinoma via targeting LDHA. Biochem Biophys Res Commun. 2018;496:947-54.

39. Lamouille S, Xu J, Derynck R. Molecular mechanisms of epithelial-mesenchymal transition. Nat Rev Mol Cell Biol. 2014;15:178-96.

40. Zhang P, Sun Y, Ma L. ZEB1: at the crossroads of epithelial-mesenchymal transition, metastasis and therapy resistance. Cell Cycle. 2015;14:481-7.

41. Tan EJ, Olsson AK, Moustakas A. Reprogramming during epithelial to mesenchymal transition under the control of TGFbeta. Cell Adhes Migr. 2015;9:233-46.

42. Mani SA, Guo W, Liao MJ, Eaton EN, Ayyanan A, Zhou AY, Brooks M, Reinhard F, Zhang CC, Shipitsin M, Campbell LL, Polyak K, Brisken C, Yang J, Weinberg RA. The epithelial-mesenchymal transition generates cells with properties of stem cells. Cell. 2008;133:704-15.

43. Flemban A, Qualtrough D. The potential role of hedgehog signaling in the luminal/basal phenotype of breast epithelia and in breast cancer invasion and metastasis. Cancers. 2015;7:1863-84.
Ready to submit your research? Choose BMC and benefit from:

- fast, convenient online submission

- thorough peer review by experienced researchers in your field

- rapid publication on acceptance

- support for research data, including large and complex data types

- gold Open Access which fosters wider collaboration and increased citations

- maximum visibility for your research: over $100 \mathrm{M}$ website views per year

At BMC, research is always in progress.

Learn more biomedcentral.com/submissions 